\title{
Implementación de ITIL versión 3 en las organizaciones: Razones del éxito y fracaso
}

\section{Implementation of ITIL version 3 in organizations: Reasons for success and failure}

Sánchez Casanova, Fiorella Stefhanie $\mathrm{e}^{1[0000-0003-0068-9250]}$ y VallesCoral, Miguel Angel $1[0000-0002-8806-2892]$

${ }^{1}$ Universidad Peruana Unión, Tarapoto, Perú. ${ }^{2}$ Universidad Nacional de San Martín, Tarapoto, Perú. fiorella.sanchez@upeu.edu.pe

Resumen. La implementación de un marco de trabajo como la Biblioteca de Infraestructura de Tecnologías de Información en las organizaciones es fundamental para una buena gestión de servicios de las tecnologías de la información. El objetivo de esta revisión fue explorar y mostrar cuáles son las razones del éxito o fracaso de una implementación ITIL versión 3. Se empleó la metodología de revisión sistemática de la literatura para la búsqueda y clasificación de estudios confiables y de calidad relacionados con el objetivo. La investigación permitió identificar 12 razones tanto de éxito como de fracaso, las cuales están relacionadas con el factor humano y la organización. Finalmente se concluyó que el éxito gira en torno al compromiso, actitud y aptitud tanto de la alta gerencia como de todas las personas involucradas en el proyecto, además de la comunicación fluida y buen clima laboral entre estas, asimismo, el fracaso se debe principalmente a la deficiente determinación de los objetivos y alineación estratégica del proyecto.

\section{Palabras clave: Calidad, información, gestión, servicio, tecnología}

Citar como: SánchezCasanova, F. S. \& VallesCoral, M. A. (2021). Implementación de ITIL versión 3 en las organizaciones: Razones del éxito y fracaso. Revista Científica De Sistemas E Informática, 1(2), 54-66. https://doi.org/10.51252/rcsi.v $1 \mathrm{i} 2.191$

Recibido: $15 / 05 / 2021$

Aceptado: 22/06/202

Publicado: 19/07/2021
Abstract. The implementation of a framework such as the Information Technology Infrastructure Library in organizations is essential for good management of information technology services. The objective of this review was to explore and show the reasons for the success or failure of an ITIL version 3 implementation. The systematic literature review methodology was used to search and classify reliable and quality studies published in bookstores. renowned digital target related. The research and review of different studies on ITIL implementation, identified 12 reasons for both success and failure, which are related to the human factor and organization, in some cases these reasons were explicitly obtained and in others it was adopted the inference method. Finally, it was concluded that success revolves around the commitment, attitude and aptitude of both senior management and all the people involved in the project, in addition to fluent communication, a good interpersonal relationship, and a good working environment between them, likewise, the failure is mainly due to the poor determination of the objectives and strategic alignment of the project. 


\section{$1 \quad$ Introducción}

A 2020, las organizaciones están enfocadas en la incorporación de las Tecnologías de la Información y las Comunicaciones (TIC) en sus procesos, esto genera la necesidad de que se identifiquen los cuellos de botella con el propósito de hacerlos más eficientes y eficaces, y dar un valor agregado a sus procesos, productos, servicios y a la vez hacerlos más competitivos (D. N. López, 2017; Puentes \& Maestre-Góngora, 2019).

$\mathrm{Si}$ bien es cierto, la influencia de las TIC es notoria, las organizaciones, tienen problemas relacionados con la inadecuada prestación de servicios de TI por el deficiente uso de las herramientas tecnológicas (Palacios-Osma et al., 2017). (da Silva et al., 2020; Eikebrokk \& Iden, 2017) consideran que es fundamental elaborar estrategias para la prestación de servicios, desarrollando e implementando una infraestructura organizativa adecuada para dichos servicios de TI, como ITIL versión 3.

ITIL o Biblioteca de Infraestructura de TI, es un modelo de referencia o framework que debe ser comprendido y utilizado para crear valor para los proveedores de servicios y usuarios (Dukić \& Babarogić, 2017). La guía de ITIL es descriptiva y está fundamentada con el ciclo de vida del servicio. La versión 3 establece un grupo de procesos, roles y funciones para el buen funcionamiento de las TI y está constituida por cinco fases o etapas que parten desde la estrategia del servicio hasta la mejora continua (C. Gómez et al., 2018; Kovácsné, 2017).

En países como Reino Unido, Alemania y España es común la implementación de ITIL, sin embargo, en la última década se fue extendiendo hasta Latinoamérica paulatina e incrementalmente pero no de igual manera en estos países, pues depende mucho del factor socioeconómico (Valle \& Ibarra, 2019).

Perú, es uno de los países latinoamericanos que ha presentado avances en la implementación de ITIL, debido a que las organizaciones se basan en las mejores prácticas con relación a la satisfacción del cliente que ofrece ITIL versión 3 (Bayona et al., 2017).

La adopción e implementación de ITIL representa un área donde la estrategia como la práctica influye en la prestación de servicios de TI y crea beneficios en muchas empresas. (Eikebrokk \& Iden, 2017) indican que, a pesar de su potencial, implementar ITIL no es sencillo, sino más bien es un reto porque numerosas organizaciones fracasan y otros siguen implementando ITIL después de la fecha prevista por los obstáculos que se presentan en el transcurso.

Las organizaciones que están interesadas en beneficiarse económicamente con ITIL, se hacen cargo de los costos que implica su adopción. Sin embargo, otras organizaciones, aún no asumen el riesgo que conlleva el proceso de implementación, ya sea porque los gerentes de TI minimizan los costos, tiempos y esfuerzos, o por la escasez de estudios que guíen sobre los factores a tener 
en cuenta para que la implementación de ITIL versión 3 sea exitosa (P. Gómez \& Salas, 2019; Mora et al., 2018).

El éxito de una implementación de ITIL versión 3 se debe principalmente a la gestión que se llegue a realizar. Los jefes de proyecto deben realizar constantes monitoreos para evaluar el progreso del proyecto, identificar riesgos y evitar estos mismos. Otro factor importante es la relación interpersonal entre los trabajadores tanto del proyecto, organización y agentes externos al proyecto. Es fundamental sostener una comunicación fluida con aquellas personas que puedan influenciar de manera positiva o negativa en el proyecto (Latorre et al., 2017; Puentes \& MaestreGóngora, 2019).

La deficiente atención al cambio organizacional, la deficiente comunicación, poco involucramiento de la alta dirección, poca importancia y priorización hacia los proyectos de TI, son algunos de los factores que llevan al fracaso una implementación de ITIL, afectando el servicio y la gestión de TI en la organización (C. Gómez et al., 2018).

Para el éxito o fracaso, un factor importante es el enfoque al servicio, ofreciendo un valor agregado y relacionando calidad con precio para el beneficio del cliente. Esta mentalidad no está presente en muchas áreas y organizaciones de TI, y no puede ser cambiada fácilmente. Si se llega a implementar sin esa seguridad del previo cambio de mentalidad, el proyecto fracasará sin dudar (Puentes \& Maestre-Góngora, 2019; Quintero \& Peña, 2017).

La presente revisión tiene como objetivo explorar y mostrar las razones que llevan al éxito o fracaso una implementación de ITIL versión 3, de manera que las empresas peruanas que decidan incursionar en esta experiencia, orienten sus acciones para evitar fracasos y pérdidas de recursos tanto en personal, tiempo y dinero.

\section{Materiales y Métodos}

Para la realización de la presente revisión y el logro del objetivo de estudio, se empleó la metodología de Revisión Sistemática de la Literatura (SLR), la cual ayudó con la clasificación de las publicaciones de acuerdo al interés y relevancia de las mismas. (Melendez-Llave \& DávilaRamón, 2018) mencionan tres pasos a seguir para que la SLR sea estricta y confiable.

\subsection{Planificación de la revisión}

En esta etapa se definió el objetivo de la investigación y se eligieron criterios de exclusión e inclusión de los trabajos de investigación. En cuanto a los criterios de exclusión, se excluyeron estudios publicados en repositorios y aquellos que no fueron publicados en revistas científicas indexadas. En criterios de inclusión, se consideraron estudios que guarden relación con el objetivo de la investigación y aquellos que estén publicados en librerías digitales de renombre. 


\subsection{Ejecución de la revisión}

Se tuvo como eje principal de búsqueda bibliográfica la metodología ITIL versión 3 y su implementación, en artículos científicos de revistas indexadas que pertenezcan a bases de datos académicas, buscadores y herramientas bibliográficas como ScienceDirect, IEEE, Scopus, Ebsco y Scielo. Asimismo, los criterios de búsqueda empleados respecto al año fueron: desde el 2017 hasta la actualidad (2020). Las palabras claves utilizadas para facilitar la búsqueda fueron: gestión, servicio, tecnología, información, calidad, ITIL, implementación, éxito, fracaso.

Después de la revisión de los diferentes estudios sobre la implementación de ITIL, se determinó las razones del éxito y fracaso de la misma, en algunos casos se obtuvieron de manera explícita y en otros se adoptó el método de la inferencia.

\subsection{Reporte de la revisión}

Esta etapa estuvo orientada al cierre formal de la investigación. Se presentó los resultados obtenidos y sintetizados por la investigadora, se analizó si dichos resultados tenían relación con el objetivo inicial, y finalmente se redactó las conclusiones. Con todo lo aprendido e investigado, la base de conocimiento fue actualizada.

\section{Resultados}

ITIL es un marco de trabajo que plantea sugerencias, alternativas y soluciones para la mejora de los servicios de TI, no se necesita implementar todos los requisitos de ITIL, debido a que no es una norma obligatoria, sino una prescripción. Surge como una recopilación de resultados alcanzados del quehacer diario por expertos en TI. En la década de los ochenta, el gobierno británico solicitó a la Agencia Central de Computación y Telecomunicaciones que establezca una guía que ayude en el uso eficaz de los activos de TI. En el año 2000, ITIL pasa por una revisión y pequeñas mejoras del marco a la que se le conoce como ITIL V2; en 2007, pasa por su segunda revisión y mejora, llegando a conocerse como ITIL V3, finalmente en 2011 se publica la última actualización de esta versión (Limanto et al., 2017; Suryawan \& Veronica, 2018). El ciclo de vida del servicio de acuerdo a ITIL versión 3 consta de cinco fases, las cuales se mencionan a continuación:

\subsection{Estrategia del Servicio}

El objetivo es identificar y analizar las necesidades del cliente para establecer una visión general de lo que se brindará en términos de servicios de TI. Esta etapa es primordial para la elaboración de planes que permita el crecimiento sostenible de la organización, razón por la que es considerada como etapa de retroalimentación, la cual guiará y soportará los servicios de TI, 
además, es importante gestionar los recursos de TI adecuados para contar siempre con los riesgos y costos asociados (Bustamante et al., 2017).

\subsection{Diseño del Servicio}

En esta fase se aseguran que los servicios y procesos nuevos o modificados, cumplan con los requerimientos cambiantes del negocio. Para transformar los objetivos estratégicos en planes operativos para perfeccionar y asegurar los niveles de los procesos, es necesario usar los principios y métodos que esta fase ofrece (Conde-Zhingre et al., 2019; Limanto et al., 2017).

\subsection{Transición del Servicio}

En esta fase se garantiza que los servicios y procesos nuevos o modificados cumplan con los requerimientos documentados en la estrategia y diseño del servicio. Se implementan aquellos procesos que estén bien organizados y orientados a la minimización de riesgos (Limanto et al., 2017; Raflesia et al., 2017).

\subsection{Operación del Servicio}

Fase que facilita la gestión de incidentes, eventos y problemas que ocurren a diario con los servicios y procesos definidos en fases anteriores. También, se encarga de dar soporte de servicios TI a los usuarios, coordinar los procesos, funciones y actividades imprescindibles para la solicitud de servicios, gestionar la infraestructura TI, y encontrar cualquier variación en los servicios TI (P. Gómez \& Salas, 2019).

\subsection{Mejora Continua del Servicio}

Esta fase es la encargada de conservar valores para los clientes a través de la evaluación y mejora continua de la calidad de los servicios y procesos (Krishnan \& Ravindran, 2018; Limanto et al., 2017).

Para elegir y adaptar mejores prácticas o estándares dentro de la organización, se tienen varias opciones, pero se tiene en cuenta las prioridades, beneficios y ventajas que se puedan obtener para tomar decisiones al respecto (Rodríguez et al., 2018).

Es primordial entender el ciclo de vida de los servicios de TI y su integración con la gestión de servicios, comprendiendo las necesidades del cliente, instaurando los niveles de calidad de servicio, evaluando la capacidad de TI, valorando los recursos de TI, evaluando el servicio y fijando procedimientos para la mejora continua (Lisanti et al., 2018; Nugraha \& Legowo, 2017). 
Antes de una implementación ITIL se debe evaluar la situación actual de la empresa para reconocer falencias en los procesos y en el cumplimiento de roles para poder tomar decisiones al respecto. Seguidamente se debe implantar un mapeo de los procesos y, por último, crear un Acuerdo de Nivel de Servicio (SLA) que posibilitará la medición de la disponibilidad de los equipos y el responsable de estos (Latorre et al., 2017).

\subsection{Razones del éxito en la implementación de ITIL versión 3}

(Müller \& de Lichtenberg, 2018) mencionan que la alta gerencia debe tener conciencia de la necesidad de adoptar una "mentalidad de servicio" para que el proyecto de implementación sea un éxito. Los ejecutivos de la organización deben ser vistos como líderes o mentores, en donde se enfatice la mejora de los recursos humanos, se conceda importancia a la moral, y se estime el consenso, la participación y el trabajo en equipo. La organización debe ser un lugar amigable en el que las personas puedan trabajar y compartir conocimientos (González-Pérez, 2017).

(Marin et al., 2018; Valencia-Duque \& Orozco-Alzate, 2017) mencionan que la organización debe ser dinámica, emprendedora, creativa para trabajar y debe fomentar la libertad de opinión entre los trabajadores. Los ejecutivos deben ser innovadores y tomadores de riesgos. El enfoque para el logro del éxito es la competitividad, el liderazgo organizacional, y el logro de metas medibles y objetivos.

(Castel, 2018; Eikebrokk \& Iden, 2017) señalan que el factor clave para el éxito de una implementación de ITIL versión 3 es la participación activa de la alta gerencia, en la que los ejecutivos a través del liderazgo continuo demuestren compromiso, alienten al equipo y ayuden a superar obstáculos. Asimismo, el compromiso organizacional con la presencia de suficientes recursos, la participación de todo el equipo y campañas de comunicación dentro de la organización significa un amplio apoyo para crear aceptación y compromiso por parte de los trabajadores.

La eficacia está relacionada con la cantidad de esfuerzo que el grupo realiza, cuanto mayor es el nivel de autoeficacia, mejor se desempeña un individuo en sus actividades. Los integrantes del proyecto deben tener habilidades para identificar, analizar y mejorar procesos, para ello, deben esforzarse al máximo y sus conocimientos sobre ITIL deben ser lo suficientes para que la implementación tenga éxito (Fragoso \& Pineda, 2018; Pérez et al., 2018).

El éxito de ITIL en las organizaciones, se debe principalmente a la actitud y aptitud de las personas, al compromiso de la alta gerencia, a la clara definición de los procesos y a la inclusión de los empleados involucrados en la toma de decisiones para transmitir la importancia de su participación (Müller \& de Lichtenberg, 2018; Pinto et al., 2017). 
El director de proyecto debe capacitarse constantemente para identificar si los indicadores sugeridos en el proyecto contribuyen al cumplimiento de las necesidades, además, las personas implicadas en el proyecto deben tener conocimientos acerca de proyectos, del desarrollo e implementación de los mismos (Ariza, 2017; C. Gómez et al., 2018).

Los resultados serán favorables, tanto para el área como para los usuarios de la empresa si se estructuran las actividades, políticas y roles, se definen indicadores como, satisfacción del cliente, productividad, tiempo de respuesta y solución a incidentes para medir la calidad del servicio, y si se hace uso de manera correcta de los servicios de ayuda para que los clientes confíen cada vez más en las empresas de TI (Quintero \& Peña, 2017).

Para el éxito de un proyecto de TI es indispensable la ayuda de las directivas, el buen entendimiento del negocio, el equipo debe estar calificado, se debe contener planes de contingencias, buenos análisis para la solución del problema presentado, enriquecer las ideas de los usuarios, compromiso por parte del personal, uso de herramientas tecnológicas, y capacidad de asumir riesgos (Peña-Casanova \& Anias-Calderón, 2020).

\subsection{Razones del fracaso en la implementación de ITIL versión 3}

Desafortunadamente no todos los casos de implementación de ITIL terminan siendo un éxito. ITIL es una nueva manera de pensar en la dirección de los servicios de TI en una organización, pero su implementación no tiene que ser vista como parte de una moda, si no se sabe el porqué es necesario, qué beneficios traerá, qué servicios de TI se ofrecerá ni qué valor se dará a las TI porque la implementación fracasará (Labanda-Jaramillo et al., 2019; Shaykhian et al., 2019). En algunos casos la implementación de ITIL, no alcanzan los resultados deseados, debido a que no se definen los objetivos de negocio, la alta dirección no participa en ella, la resistencia al cambio, la falta de motivación y la falta de comunicación entre los miembros del equipo (ArcillaCobian et al., 2017; Parra, 2018).

Luego de una implementación ITIL, los problemas de servicios de TI pueden continuar y no notarse ninguna mejoría, si tanto la empresa como el equipo toman este proyecto de ITIL como uno más del área de sistemas, si no se le da la importancia debida y si no se estima el enorme impacto que tiene ITIL en la entrega de valor de los servicios de TI a los clientes finales (Ariza, 2017; Bayona et al., 2017).

(Dukić \& Babarogić, 2017) manifiestan que si la organización se centra solo en estandarizar el comportamiento comercial para que el esfuerzo y trabajo de cada trabajador fuera apreciado adecuadamente, se tendrán problemas en la implementación porque el control de calidad en el departamento de TI es escaso, la comunicación entre los trabajadores no está del todo definida, 
existe cantidad de tareas repetitivas, los objetivos son irrazonables, existe mala distribución del trabajo porque algunos trabajadores están sobrecargados y otros poco.

La implementación fracasará si las expectativas del proyecto son confusas debido a que las necesidades del cliente no son entendidas en su totalidad y el proyecto es descrito y planificado como algo pequeño, cuando en realidad puede llegar a ser más complejo y emplear más tiempo y recursos (Dukić \& Babarogić, 2017; C. López, 2018).

El cambio de personal clave en el proyecto es una de las principales causas de fracaso y retraso del mismo. Igualmente, el proyecto fracasará, si inicialmente no se define una buena matriz de roles y responsabilidades, no se estiman bien los tiempos ni se seleccionan los recursos claves que el proyecto de ITIL necesitará (González-Pérez, 2017).

La falta de experiencia del personal de TI implicado en el proyecto de implementación puede llegar a ser perjudicial para el progreso del mismo, por lo que es fundamental para el director del proyecto identificar los conocimientos y habilidades de los miembros del equipo, y a partir de ello decidir si es necesario una charla o capacitación sobre ITIL (C. Gómez et al., 2018; Guzmán et al., 2018).

(Melendez-Llave \& Dávila-Ramón, 2018; Parra, 2018) mencionan que una implementación fracasa por el limitado apoyo de la alta gerencia para afrontar las dificultades que se presenten, por la escasa planeación y determinación de los objetivos, por los escasos monitoreos del proyecto, y por la descoordinación y falta de comunicación entre los procesos, tecnologías y personas. Asimismo, otro factor del fracaso es la resistencia al cambio de los trabajadores, quiénes responden a los cambios lentamente.

Luego de recolección y análisis de la información, se pudo determinar que las razones que llevan al éxito o al fracaso una implementación de ITIL versión 3 son las siguientes (ver Tabla 1): 
Tabla 1

Razones del éxito y fracaso de Implementación de ITIL v3.

\begin{tabular}{ll}
\hline \multicolumn{1}{c}{ Implementación ITIL versión 3 } \\
\hline Razones del éxito & Razones del fracaso \\
\hline Compromiso de la alta gerencia. & No se determinan los objetivos del negocio. \\
$\begin{array}{l}\text { Buen entendimiento del negocio. } \\
\text { Definición de procesos. }\end{array}$ & $\begin{array}{l}\text { Limitada colaboración de la alta dirección. } \\
\text { Insuficiente planeación del proyecto. }\end{array}$ \\
$\begin{array}{l}\text { Actitud, aptitud y compromiso de las personas } \\
\text { involucradas. }\end{array}$ & Deficiente alineación estratégica del negocio. \\
$\begin{array}{l}\text { Monitoreos constantes para evaluar el progreso del } \\
\text { proyecto. }\end{array}$ & $\begin{array}{l}\text { Desestimación del impacto de ITIL en la entrega de valor } \\
\text { de los servicios de TI a los clientes finales. }\end{array}$ \\
$\begin{array}{l}\text { Comunicación fluida y buena relación interpersonal } \\
\text { entre los trabajadores tanto del proyecto, organización }\end{array}$ & Poca importancia hacia el proyecto de ITIL. \\
y agentes externos al proyecto. & \\
$\begin{array}{l}\text { Constante capacitación del director de proyecto. } \\
\text { Objetivos del proyecto bien identificados. }\end{array}$ & $\begin{array}{l}\text { Poca priorización hacia el proyecto de ITIL. } \\
\text { Existencia de planes de contingencia. }\end{array}$ \\
$\begin{array}{l}\text { Poca comunicación de las partes implicadas. } \\
\text { Asumir riesgos. }\end{array}$ & $\begin{array}{l}\text { Personas poco capacitadas y sin experiencia en proyecto } \\
\text { de TI. }\end{array}$ \\
$\begin{array}{l}\text { Liderazgo organizacional } \\
\text { Eficacia del personal }\end{array}$ & $\begin{array}{l}\text { Poca participación y poco compromiso } \\
\text { Inexperiencia técnica del personal de TI. }\end{array}$ \\
\hline
\end{tabular}

Como se observa en la anterior tabla, el éxito se debe principalmente al análisis de selección de buenas prácticas que recomienda este marco de trabajo para la satisfacción de los clientes y/o usuarios finales y la entrega de servicios de manera adecuada. Texto. Nos mencionan una figura, ecuación o fórmula.

\section{Conclusiones}

La revisión permitió identificar que el éxito se debe principalmente a la motivación, inclusión y compromiso durante todo el proyecto de las personas que serán impactadas por la implementación de ITIL v3, asimismo, es importante mantener el buen clima laboral y una buena comunicación entre las partes.

Las razones principales del fracaso son la deficiente determinación de los objetivos, la inadecuada alineación estratégica y la poca importancia del proyecto que están relacionados con la falta de gestión y liderazgo por parte de la alta dirección, debido a que no tienen una visión general del proyecto y es por eso que la toma de decisiones no siempre es efectiva.

El éxito o fracaso dependerá de cómo los involucrados de la implementación se adapten a los retos y cambios que se presenten para el logro de sus objetivos. Si se pierde el control del tiempo, costo y alcance, el proyecto fracasará indiscutiblemente por eso la clave para que esto no suceda es mantener el equilibrio de estos 3 factores. 


\section{Referencias bibliográficas}

Arcilla-Cobian, M., San Feliu, T., Feliz, A., \& Calvo-Manzano, J. A. (2017). Implementación de una Biblioteca de Activos de Proceso orientada a la Gestión de la Capacidad de Servicios de TI. Iberian Conference on Information Systems and Technologies, CISTI, 4(2), 43-51. https://doi.org/10.23919/CISTI.2017.7975763

Ariza, D. A. (2017). Efectividad de la gestión de los proyectos: una perspectiva constructivista. Obras y proyectos, 22, 75-85. https://doi.org/10.4067/s0718-28132017000200075

Bayona, S., Baca, Y., \& Vela, G. (2017). Gestión de Servicios de TI Usando ITIL v3: Caso de Estudio. Iberian Conference on Information Systems and Technologies, CISTI, 26-32. https://doi.org/10.23919/CISTI.2017.7975963

Bustamante, F., Fuertes, W., Diaz, P., \& Toulkeridis, T. (2017). Integration of IT frameworks for the management of information security within industrial control systems providing metrics and indicators. Proceedings of the 2017 IEEE 24th International Congress on Electronics, Electrical Engineering and Computing, INTERCON 2017, 15-18. https://doi.org/10.1109/INTERCON.2017.8079672

Castel, A. F. (2018). La integración de las TIC en los procesos educativos y organizativos A integração das TIC nos processos educacionais e organizativos. Educar em Revista, 34(69), 325-339. https://doi.org/10.15900104-4060.57305

Conde-Zhingre, L. E., Quezada-Sarmiento, P. A., \& Hernandez, W. (2019). Propuesta de Arquitectura de mesa de servicios tecnológicos basado en el marco de referencia ITIL V 3.0. Iberian Conference on Information Systems and Technologies, CISTI, 2019June(June), 19-22. https://doi.org/10.23919/CISTI.2019.8760832

Da Silva, H. C., Silva, D., Dornelas, J. S., \& Ferreira, H. S. (2020). Information technology governance in small and medium enterprises - a Systematic Mapping. Jistem - Journal of Information Systems and Technology Management, 17. https://doi.org/10.4301/s18071775202017001

Dukić, N., \& Babarogić, S. (2017). Upravljanje it uslugama u državnom organu primenom itil preporuka it service management in government body using ITIL recommendations. InfoM-Journal of Information Technology and Multimedia Systems, 62, 10-16. http://web.b.ebscohost.com/ehost/detail/detail?vid=2\&sid=34bc9129-c4e4-424e-a2d800ce0a779d49\%40pdc-vsessmgr02\&bdata=Jmxhbmc9ZXMmc210ZT1laG9zdC1 saXZl\#AN=124121663\&db=iih

Eikebrokk, T. R., \& Iden, J. (2017). Strategising IT service management through ITIL implementation: model and empirical test. Total Quality Management and Business Excellence, 28(3-4), 238-265. https://doi.org/10.1080/14783363.2015.1075872

Fragoso, H., \& Pineda, D. (2018). Modelo de gobierno de tecnología de la información para 
mejorar el desempeño de proyectos de negocio minorista. Investigación administrativa, 47(22). http://www.scielo.org.mx/pdf/ia/v47n122/2448-7678-ia-47-122-00004.pdf

Gómez, C., Valencia, F., \& Marulanda, C. (2018). Las Tecnologías de la Información y las Comunicaciones y los Servicios Tecnológicos en las Entidades Públicas del Triángulo del Café en Colombia. Información Tecnológica, 29(4), 119-128. https://doi.org/10.4067/s0718-07642018000400119

Gómez, P., \& Salas, R. (2019). Prestación del servicio de producción de tecnología educativa con base en las buenas prácticas de la librería ITIL. RIDE - Revista Iberoamericana para la Investigación y el Desarrollo Educativo, 9(18), 683-716. http://www.scielo.org.mx/pdf/ride/v9n18/2007-7467-ride-9-18-683.pdf

González-Pérez, A. (2017). Dinamización tecnológica de la escuela a través del liderazgo del coordinador TIC. Estudios Pedagógicos, 43(2), 115-125. https://doi.org/10.4067/S071807052017000200006

Guzmán, B. A., Jiménez, M., \& Hernández, I. A. (2018). Medición de la gestión del conocimiento en la Universidad Pública de la Ciudad de México / Measurement of knowledge management at the public university at Mexico city. RIDE Revista Iberoamericana para la Investigación y el Desarrollo Educativo, 9(17), 604-622. https://doi.org/10.23913/ride.v9i17.398

Kovácsné, A. L. M. (2017). Reducing it costs and ensuring safe operation with application of the portfolio management. Serbian Journal of Management, 12(1), 143-155. https://doi.org/10.5937/sjm12-11452

Krishnan, G., \& Ravindran, V. (2018). IT service management automation and its impact to IT industry. ICCIDS 2017 - International Conference on Computational Intelligence in Data Science, Proceedings, 2018-Janua, 5-8. https://doi.org/10.1109/ICCIDS.2017.8272633

Labanda-Jaramillo, M., Chamba-Eras, L., Coronel-Romero, E., Granda, J., Quezada-Sarmiento, P., \& Roman-Sanchez, M. (2019). Propuesta de Reingeniería de los Procesos en la Gestión de las Tecnologías de la Información y Comunicación. Iberian Conference on Information Systems and Technologies, CISTI, 2019-June(June), 19-22. https://doi.org/10.23919/CISTI.2019.8760700

Latorre, J., Fiallo, O., Díaz, C., \& Chacon, J. (2017). Propuesta metodológica para analizar y evaluar los riesgos presentados en el proceso de contratación en proyectos tecnológicos. Frónesis, 24(3). http://web.b.ebscohost.com/ehost/pdfviewer/pdfviewer?vid=0\&sid=9a4a24d5-dbfa-47448a59-627b907aa8c9\%40sessionmgr101

Limanto, A., Khwarizma, A., Rumagit, R., Pietono, V., Halim, Y., \& Liawatimena, S. (2017). A study of Information Technology Infrastructure Library (ITIL) framework implementation at the various business field in Indonesia. 2017 th International Conference on Cyber and 
IT Service Management, CITSM 2017, 1-4. https://doi.org/10.1109/CITSM.2017.8089244

Lisanti, Y., Luhukay, D., \& Mariani, V. (2018). IT service and risk management implementation for online startup SME: Case study: Online startup SME in Jakarta. Proceedings of 2017 International Conference on Information Management and Technology, ICIMTech 2017, 2018-January(November), 300-303. https://doi.org/10.1109/ICIMTech.2017.8273555

López, C. (2018). Guía Técnica-Metodológica de Definición de Proyectos de TIC's, una visión con enfoque en el modelo de 8 pilares para la gestión. Revista Cubana de Ciencias Informáticas, 12, 119-134. http://scielo.sld.cu/pdf/rcci/v12s1/rcci09518.pdf

López, D. N. (2017). Modelo de gestion de los servicios de tecnologia de informacion basado en COBIT, ITIL e ISO/IEC 27000. Revista Tecnológica - ESPOL, 30(1), 51-69. http://www.scielo.org.mx/pdf/prts/v8n14/2007-3607-prts-8-14-00003.pdf

Marin, A., Trujillo, Y., \& Buedi, D. (2018). Framework to manage software quality activities. Revista Cubana de Ciencias Informáticas, 12(2), 74-88. http://scielo.sld.cu/pdf/rcci/v12n2/rcci06218.pdf

Melendez-Llave, K. A., \& Dávila-Ramón, A. E. (2018). Problemas en la adopción de modelos de gestión de servicios de tecnologías de información. Una revisión sistemática de la literatura. Dyna, 85(204), 215-222. https://doi.org/10.15446/dyna.v85n204.57076

Mora, M., Castillo, M., Muñoz, R., \& Salas, J. (2018). Despliegue de ITIL como marco de buenas prácticas en las empresas de equipamiento e integración de servicios de video conferencia en Chile y el mundo. Revista Científica de la UCSA, 5(1), 61-72. https://doi.org/10.18004/ucsa/2409-8752/2018.005(01)061-072

Müller, S. D., \& De Lichtenberg, C. G. (2018). The culture of ITIL: Values and implementation challenges. Information Systems Management, 35(1), 49-61. https://doi.org/10.1080/10580530.2017.1416946

Nugraha, A., \& Legowo, N. (2017). Implementation of incident management for data services using ITIL V3 in telecommunication operator company. Proceedings - 2017 International Conference on Applied Computer and Communication Technologies, ComCom 2017, 2017-January, 1-6. https://doi.org/10.1109/COMCOM.2017.8167093

Palacios-Osma, J., Rodríguez-Guzmán, J. L., \& García-Ramírez, C. (2017). Modelo de gestión de servicios ITIL para E-learning 1 ITIL services management model for E-learning. Revista Educación en Ingeniería. Febrero, 12(23), 28-34. https://doi.org/10.26507/rei.v12n23.684

Parra, J. E. (2018). La gestión del conocimiento en la planificación y desarrollo de proyectos informáticos. Revista Cubana de Ciencias Informáticas, 12, 105-118. http://scielo.sld.cu/pdf/rcci/v12s1/rcci08518.pdf

Peña-Casanova, M., \& Anias-Calderón, C. (2020). Integración de marcos de referencia para 
gestión de Tecnologías de la Información. Ingeniería Industrial, 41(1), 1-12. http://scielo.sld.cu/pdf/rii/v41n1/1815-5936-rii-41-01-e4110.pdf

Pérez, L., Carrera, J., \& García, A. M. (2018). Eficacia como constructo multidimensional en la determinación de estrategias de informatización empresarial. Ingeniare. Revista chilena de ingeniería, 26(2), 354-369. https://doi.org/10.4067/s0718-33052018000200354

Pinto, S. M., Ramírez, P. E., \& Grandón, E. E. (2017). Antecedentes del Éxito de los sistemas de planificación de recursos empresariales en las grandes empresas chilenas: Un modelo factorial exploratorio. Información Tecnológica, 28(3), 139-146. https://doi.org/10.4067/S0718-07642017000300015

Puentes, C. E., \& Maestre-Góngora, G. P. (2019). Plan estratégico basado en ITIL para mipymes en el departamento de Arauca-Colombia. Lámpsakos, 22, 68-84. https://doi.org/10.21501/21454086.3280

Quintero, L., \& Peña, H. (2017). Modelo basado en ITIL para la Gestión de los Servicios de TI en la Cooperativa de Caficultores de Manizales. Scientia et Technica, 22(4), 371-380. https://doi.org/10.22517/23447214.13211

Raflesia, S., Surendro, K., \& Passarella, R. (2017). The user engagement impact along information technology of infrastructure library (ITIL) adoption. ICECOS 2017 Proceeding of 2017 International Conference on Electrical Engineering and Computer Science: Sustaining the Cultural Heritage Toward the Smart Environment for Better Future, 184-187. https://doi.org/10.1109/ICECOS.2017.8167130

Rodríguez, J. A., López, M. C., \& De Los Monteros, A. (2018). Estudio sobre la implementación del software Help Desk en una institución de educación superior. PAAKAT: Revista de Tecnología y Sociedad, 8(14), 3. https://doi.org/10.18381/pk.a8n14.298

Shaykhian, G., Said, K. A., \& Alqarna, G. (2019). Itil its effectiveness: Decision makers' perspectives. ASEE Annual Conference and Exposition, Conference Proceedings. http://web.a.ebscohost.com/ehost/detail/detail?vid=0\&sid=95c07e89-17b3-4197-97befe105d0faa48\%40sdc-vsessmgr01\&bdata=Jmxhbmc9ZXMmc210ZT1laG9zdC1 saXZl\#AN=139582436\&db=a9h Suryawan, A. D., \& Veronica. (2018). Information Technology Service Performance Management Using COBIT and ITIL Frameworks : A Case Study. Proceedings of 2018 International Conference on Information Management and Technology, ICIMTech 2018, September, 223-228. https://doi.org/10.1109/ICIMTech.2018.8528197

Valencia-Duque, F. J., \& Orozco-Alzate, M. (2017). Metodología para la implementación de un Sistema de Gestión de Seguridad de la Información basado en la familia de normas ISO/IEC 27000. RISTI - Revista Iberica de Sistemas e Tecnologias de Informacao, 22, 7388. https://doi.org/10.17013/risti.22.73-88 
Valle, R., \& Ibarra, Y. (2019). La calidad asociada al proceso de gestión de riesgos. Revista Antioqueña de las Ciencias Computacionales y la Ingeniería de Software (RACCIS), 9(1), 28-36. https://doi.org/10.5281/zenodo.3260323

\section{Conflicto de intereses}

No existe ningún conflicto de intereses para la publicación del presente artículo.

\section{Contribuciones de los autores}

Fiorella Stefhanie Sánchez Casanova, investigadora realizó el diseño, búsqueda, análisis e interpretación de la información para la redacción del artículo. Miguel Ángel Valles Coral, investigador y facilitador del presente artículo. 